\title{
Collisionless reconnection: mechanism of self-ignition in thin plane homogeneous current sheets
}

\author{
R. A. Treumann ${ }^{1,2,{ }^{*}}$, R. Nakamura ${ }^{3}$, and W. Baumjohann ${ }^{3}$ \\ ${ }^{1}$ Department of Geophysics and Environmental Sciences, Munich University, Munich, Germany \\ ${ }^{2}$ Department of Physics and Astronomy, Dartmouth College, Hanover NH 03755, USA \\ ${ }^{3}$ Space Research Institute, Austrian Academy of Sciences, Graz, Austria \\ *currently at: International Space Science Institute, Bern, Switzerland
}

Received: 25 July 2010 - Accepted: 20 October 2010 - Published: 26 October 2010

\begin{abstract}
The spontaneous onset of magnetic reconnection in thin plane collisionless current sheets is shown to result from a thermal-anisotropy driven non-relativistic magnetic electron Weibel-mode, generating seed-magnetic field $\mathrm{X}$-points in the centre of the current layer. The proposed mechanism is of larger generality. It also works in the presence of magnetic guide fields.
\end{abstract}

Keywords. Magnetospheric physics (Storms and substorms) - Space plasma physics (Magnetic reconnection)

\section{Introduction}

The idea of magnetic reconnection as the main plasma process that converts stored magnetic energy into kinetic energy originates from the intuitive geometric picture of annihilating antiparallel magnetic field lines when approaching each other (see, e.g., Sweet, 1957; Parker, 1958; Dungey, 1961). Observations in space have unambiguously confirmed the presence of reconnection under collisionless conditions (see, e.g., Fujimoto et al., 1997; Øieroset et al., 2001; Nagai et al., 2001) when the fluid theoretical approaches break down. However, no convincing theoretical argument for the spontaneous occurrence of reconnection has so far been given. In most collisionless numerical simulations reconnection is artificially ignited (cf., e.g., Zeiler et al., 2000), mostly by ad hoc imposing a seed X-point on the current sheet separating the anti-parallel fields. (Imposing a seed-X-point has a number of effects which we will briefly discuss in the final

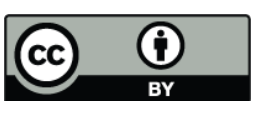

Correspondence to: R. A. Treumann (rudolf.treumann@geophysik.unimuenchen.de) discussion section.) The ongoing search for the mechanism of spontaneous onset of collisionless reconnection points to the "missing microphysics" in thin current sheets. The exception is a paper by Ricci et al. (2004) where reconnection is started from scratch and is attributed to the action of the lower-hybrid drift instability thereby referring to diffusive driving of reconnection.

In the present Letter we show that instability of the inner current layer gives rise to the self-consistent generation of local magnetic fields $\boldsymbol{B}=\left(B_{\mathrm{x}}, 0, B_{\mathrm{Z}}\right)$ transverse to the current layer. Such local fields are equivalent to the generation of microscopic seed X-points in the current sheet centre and are capable of spontaneously igniting reconnection as is known from two-dimensional PIC particle simulations (cf., e.g., Zeiler et al., 2002; Scholer et al., 2003, and others). Since, in an ideal current sheet, ions and electrons become non-magnetic on their respective inertial scales $\lambda_{\mathrm{i}, \mathrm{e}}=c / \omega_{\mathrm{i}, \mathrm{e}}$, where $\omega_{\mathrm{i}, \mathrm{e}}=e \sqrt{N / \epsilon_{0} m_{\mathrm{i}, \mathrm{e}}}$ are the plasma frequencies of ions and electrons, (classical) collisionless convective transport of magnetic fields into the current layer takes place up to a vertical distance $z \sim \lambda_{\mathrm{e}}$ from the centre of the current sheet. The region between $\lambda_{\mathrm{e}} \lesssim z \lesssim \lambda_{\mathrm{i}}$ is known as the "Hall-current" (Sonnerup, 1979) or (mistakenly, as there is no diffusion present) "ion-diffusion" region. They close along the magnetic field by electrons that are accelerated in the oblique lower-hybrid-drift/modified-two-stream instability driven by magnetised Hall-electrons on the non-magnetic ion background thereby coupling the reconnection site to the auroral ionosphere (Treumann et al., 2009).

When speaking of a current sheet, we refer to ideal homogeneous current sheets separating strictly antiparallel magnetic fields thereby excluding any residual normal magnetic field components. Indeed, the observational paradigm

Published by Copernicus Publications on behalf of the European Geosciences Union. 
of a reconnecting current sheet is the magnetospheric tailcurrent sheet. This current sheet is not ideal in the above sense as it is embedded into a quasi-dipolar field which still might preserve a weak rudimentary (normal) magnetic field component $B_{\mathrm{Z}}$ pointing northward. This $B_{\mathrm{Z}}$ component remagnetises the central-sheet electrons and affects the evolution of (collisionless) tearing modes (Galeev and Zelenyi, 1975). Nevertheless below, when using numbers, we will for reasons of resolution refer to conditions in the magnetotail even though our theory might apply better to the magnetopause, interplanetary space or astrophysics. In principle, observation of the electron-inertial ("electron-diffusion") region is difficult because of its narrow width. Unambiguous observations do not yet exist. At the magnetopause, in particular, very narrow electron layers have sometimes been reported assuming that they relate to the electron-inertial region during reconnection (for a recent discussion of the experimental prospects of resolving the electron-inertial region cf., Scudder et al., 2008).

\section{Magnetic field generation in the current layer}

Unless a guide field is imposed from the outside on a plane collisionless homogeneous current sheet, the inner region $z \lesssim$ few $\lambda_{\mathrm{e}}$ of the current sheet is about free of magnetic fields, while at the same time carries a (diamagnetic) current $J_{\perp}$ perpendicular to the antiparallel magnetic fields to both sides of the current, caused (for instance in the geomagnetic tail current sheet or the Earths magnetopause) by a (macroscopic) electric potential drop $\Delta U$ along the current.

For the understanding of the mechanism of reconnection it is of no interest how this potential drop is generated. This may happen when two magnetised collisionless plasmas of finite lateral extension collide. In the magnetotail current sheet the potential is imposed from the outside by the solar wind and the potential amounts to $1 \lesssim \Delta U \lesssim$ few $10 \mathrm{kV}$, and electron and ion temperatures are of the order of $T_{\mathrm{e}} \sim 0.1 \mathrm{keV}$ and $T_{\mathrm{i}} \sim 1 \mathrm{keV}$, respectively. Electrons entering the centre of the current sheet accelerate along the current, thereby becoming the main current carriers here. Their high translational velocity $V_{\mathrm{e}}=\sqrt{e \Delta U / m_{\mathrm{e}}}>v_{\mathrm{e}}$ exceeds their thermal speed $v_{\mathrm{e}}=\sqrt{2 T_{\mathrm{e}} / m_{\mathrm{e}}}$ providing conditions that are unstable against the Buneman two-stream instability (Buneman, 1958), a fast growing electrostatic instability with high frequency $\omega_{\mathrm{B}} \sim$ $0.03 \omega_{\mathrm{e}}$ and large growth rate $\gamma_{\mathrm{B}} \sim \omega_{\mathrm{B}}$ (cf., e.g., Treumann and Baumjohann, 1997, p. 22). In the geomagnetic tail current sheet the growth rate amounts to $\gamma_{\mathrm{B}} \approx 1.7 \mathrm{kHz}$, corresponding to a growth time of $\tau_{\mathrm{B}} \sim 0.006 \mathrm{~s}$.

The Buneman instability readily generates localised micro-scale (several Debye lengths) electrostatic structures (known as electron and ion phase space holes) which trap a substantial part of the electrons and heat them in the direction along the current drift velocity. Numerical simulations suggest that this process takes roughly $100-1000$ plasma periods
(Buneman, 1959; Newman et al., 2001), or few 10 e-folding times, in the magnetospheric tail $\lesssim 0.1 \mathrm{~s}$. In this process the instability shuts off itself by increasing the parallel electron temperature until $v_{\mathrm{e} \|} \sim V_{\mathrm{e}}$. At the end of this very fast process the electrons develop a temperature anisotropy

$A=T_{\mathrm{e} \|} / T_{\mathrm{e} \perp}-1>0$

with current-parallel temperature $T_{\mathrm{e} \|}>T_{\mathrm{e} \perp}=T_{\mathrm{e}}$ exceeding the initial electron temperature, roughly $A \lesssim 1$ in the magnetospheric tail current sheet. The subscripts $\|$ and $\perp$ refer to the respective directions of maximum and minimum electron temperatures, i.e. the two directions of the electron pressure tensor

$$
\mathrm{P}_{\mathrm{e}}=N\left[T_{\mathrm{e} \perp} \mathrm{I}+\left(T_{\mathrm{e} \|}-T_{\mathrm{e} \perp}\right) \boldsymbol{V}_{\mathrm{e}} \boldsymbol{V}_{\mathrm{e}} / V_{\mathrm{e}}^{2}\right]
$$

In this thermally anisotropic case the electrons obey a biMaxwellian equilibrium distribution function

$f_{\mathrm{e}}\left(v_{\perp}, v_{\|}\right)=\frac{\left(m_{\mathrm{e}} / 2 \pi\right)^{\frac{3}{2}}}{T_{\mathrm{e} \perp} \sqrt{T_{\mathrm{e} \|}}} \exp \left[-\frac{m_{\mathrm{e}} v_{\perp}^{2}}{2 T_{\mathrm{e} \perp}}-\frac{m_{\mathrm{e}} v_{\|}^{2}}{2 T_{\mathrm{e} \|}}\right]$

which, in a nonmagnetised plasma (like the inner current region $z \lesssim \lambda_{\mathrm{e}}$ ) is unstable with respect to the family of Weibel ${ }^{1}$ instabilities (Weibel, 1959). These are very low (about zero) frequency (purely growing) electromagnetic instabilities which are capable of generating stationary magnetic fields that grow from thermal fluctuations (not requiring any magnetic dynamo mechanism). The linear electromagnetic dispersion relation of the plasma becomes

$\left(n^{2}-\epsilon_{\perp}\right)^{2} \epsilon_{\ell}=0$

where $n=k c / \omega$ is the refraction index, and $\omega$ is the frequency of the linear disturbance. The dielectric tensor has the two scalar components $\epsilon_{\ell}(\boldsymbol{k}, \omega), \epsilon_{\perp}(\boldsymbol{k}, \omega)$ which are the longitudinal and transverse response functions, respectively. For our purposes it suffices to consider the electromagnetic (transverse) response buried in

$\epsilon_{\perp}=1-\frac{\omega_{\mathrm{e}}^{2}}{\omega^{2}}\{1-(A+1)[1+\zeta Z(\zeta)]\}-\frac{\omega_{i}^{2}}{\omega^{2}}=n^{2}$

where $Z(\zeta)$ is the plasma dispersion function, $\zeta=\omega / k_{\perp} v_{\mathrm{e} \perp}$, and $v_{\mathrm{e} \perp}=\sqrt{2 T_{\perp} / m_{\mathrm{e}}}$ is the electron thermal speed perpendicular to the current. The Weibel instability grows in the plane perpendicular to the direction of the higher thermal velocity, which in our case has been assumed as the parallel direction. Hence, $\boldsymbol{k}=\left(k_{\mathrm{x}}, 0, k_{\mathrm{z}}\right)=\left(k_{\perp} \sin \theta, 0, k_{\perp} \cos \theta\right)$; in an extended medium there is no $\theta$-dependence, a point to which we will return later. The contribution of the resting ions has been retained for completeness; because of the smallness of the ion plasma frequency $\omega_{\mathrm{i}} \ll \omega_{\mathrm{e}}$, being much less than the

\footnotetext{
${ }^{1}$ Weibel - or current filamentation instabilities, as they are sometimes called following Fried (1959) where a simple physical model of their mechanism was given early - have mostly been investigated in view of astrophysical applications in a relativistic approach.
} 
electron plasma frequency $\omega_{\mathrm{e}}$, it plays no role in the instability.

At zero real frequency $\omega=i \gamma$ and $A>0$ the righthand side of Eq. (5) becomes the dispersion relation of the thermal-anisotropy driven Weibel mode (Weibel, 1959; Yoon and Davidson, 1987, and others). Instability $\gamma\left(k_{\perp}\right)>0$ sets on at phase velocities $\omega / k_{\perp} \ll v_{\mathrm{e} \perp}$ for wavenumbers $k_{\perp}<k_{0}$,

$k_{0} \lambda_{e} \simeq \sqrt{A}$

with instability growth rate

$\frac{\gamma_{\mathrm{W}}}{\omega_{\mathrm{e}}} \simeq \sqrt{\frac{2}{\pi}} \frac{v_{\mathrm{e} \perp}}{c} \frac{k_{\perp}}{k_{0}}\left(1-\frac{k_{\perp}^{2}}{k_{0}^{2}}\right)(A+1)\left(k_{0} \lambda_{\mathrm{e}}\right)^{3}$

vanishing at long wavelengths $k_{\perp}=0$. The growth rate maximises at wavenumber $k_{\perp \mathrm{m}}=k_{0} / \sqrt{3}=\lambda_{\mathrm{e}}^{-1} \sqrt{A / 3}$ (see Fig. 1) where its value is

$$
\frac{\gamma_{\mathrm{W}, \mathrm{m}}}{\omega_{\mathrm{e}}} \simeq \frac{4}{3} \sqrt{\frac{A^{3} \Theta_{\mathrm{e}}}{3 \pi}}(A+1)
$$

with $\Theta_{\mathrm{e}} \equiv T_{\mathrm{e} \perp} / m_{\mathrm{e}} c^{2}$ the (ambient) temperature normalised to the rest energy of an electron. Numerically this expression yields for the maximum growth rate

$\gamma_{\mathrm{W}, \mathrm{m}} \approx 34 \sqrt{N_{\left[\mathrm{cm}^{-3}\right]} T_{\mathrm{e} \perp[\mathrm{eV}]}} A^{\frac{3}{2}}(A+1) \mathrm{Hz}$

Depending on the value of the anisotropy, this growth rate can be substantial. If $A>1$, it grows as $\gamma \propto \sqrt{A^{5}}$, while for anisotropies $A<1$ it grows like $\gamma \propto \sqrt{A^{3}}$. In the tail plasma sheet we have $T_{\mathrm{e}} \sim 100 \mathrm{eV}$ and $N \sim 1 \mathrm{~cm}^{-3}$. Then, even with $A \sim 0.1$ one finds quite a fast growth rate of $\gamma_{\mathrm{W}, \mathrm{m}} \lesssim 10 \mathrm{~Hz}$.

The important point is that even though the growth rate might not be extraordinarily large, it generates a magnetic field that has two components, $\boldsymbol{B}_{\mathrm{W}}=\left(B_{\mathrm{x}}, 0, B_{\mathrm{Z}}\right)$, both being transverse to the initial current. The component $B_{\mathrm{X}}$ is alternating between the directions parallel and antiparallel to the initial magnetic field outside the current layer, being directed $\pm \hat{x}$ while the other component is perpendicular to the current layer directed along $\pm \hat{z}$. This field modulates the current layer along $\hat{x}$ causing magnetic islands whose vertexes lie in the centre of the current layer. It thus provides seed$X$ points which, if sufficiently large amplitude, will spontaneously ignite reconnection. The finite nonmagnetic current sheet width in $z$ imposes a limit $2 \pi / k_{\mathrm{z}}<2 \lambda_{\mathrm{e}}$ which yields

$k_{\mathrm{z}} / k_{\mathrm{x}}=\cot \theta \approx k_{\mathrm{z}} / k_{\mathrm{m}}>\pi \sqrt{3 / A}$

the lower limit resulting from the restriction on $A>3 m_{\mathrm{e}} / 2 m_{\mathrm{i}}$ (see below). Thus the Weibel mode propagates at angles

$\tan ^{-1}\left[\pi^{-1} \sqrt{m_{\mathrm{e}} / 2 m_{\mathrm{i}}}\right]<\theta<\tan ^{-1}\left[\pi^{-1} \sqrt{A / 3}\right]$

against $\hat{x}$. This is the maximum angle the wavevector assumes in the Weibel-field vertexes. For $A=0.1$ and $A=1$

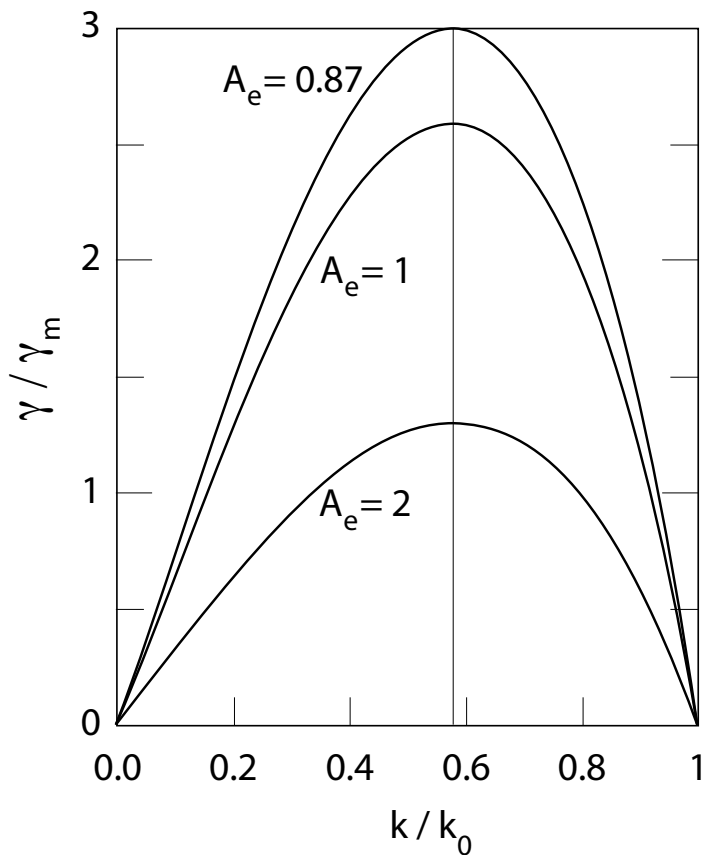

Fig. 1. The anisotropic-thermal Weibel instability growth rate $\gamma / \gamma_{m}$, normalised to maximum growth, as function of the normalised wavenumber $k / k_{0}$ for three different thermal anisotropies. This ratio increases as $A^{-1}$. The vertical line indicates the position of the maximum growing wave number $k_{m} / k_{0}$.

these inclination angles are $0.3^{\circ}<\theta \lesssim 3.4^{\circ}$ and $\sim 11^{\circ}$, respectively. However, in addition, the Weibel mode can propagate in two directions $\pm \hat{x}$. The two cases are shown in Fig. 6: (a) when the propagation direction choses to be along the external field. In this case simple seed-X points in the current sheet are generated which will allow reconnection to evolve in the usual way. For the oppositely directed Weibel vertices shown in Fig. 6 (b), however, a multitude of additional reconnection sites are produced along $z \sim \pm \lambda_{\mathrm{e}}$, and the current layer becomes highly unstable. Which is the most probable case can be decided only after a complete solution of the Weibel-unstable boundary value problem of the current layer.

\section{Thermal fluctuation level}

In order to infer how long it takes the instability to achieve substantial magnetic field amplitudes we need to estimate the magnetic thermal fluctuation level $\left\langle b_{i} b_{j}\right\rangle_{k, \omega=0}$ from where the Weibel instability starts growing in the presence of the electron pressure anisotropy (thermally fluctuating magnetic fields will be denoted by lower case letters). Magnetic thermal levels have recently been estimated (Yoon, 2007b; Baumjohann et al., 2010). From basic fluctuation theory (Sitenko, 1967) the spectral energy density of the 


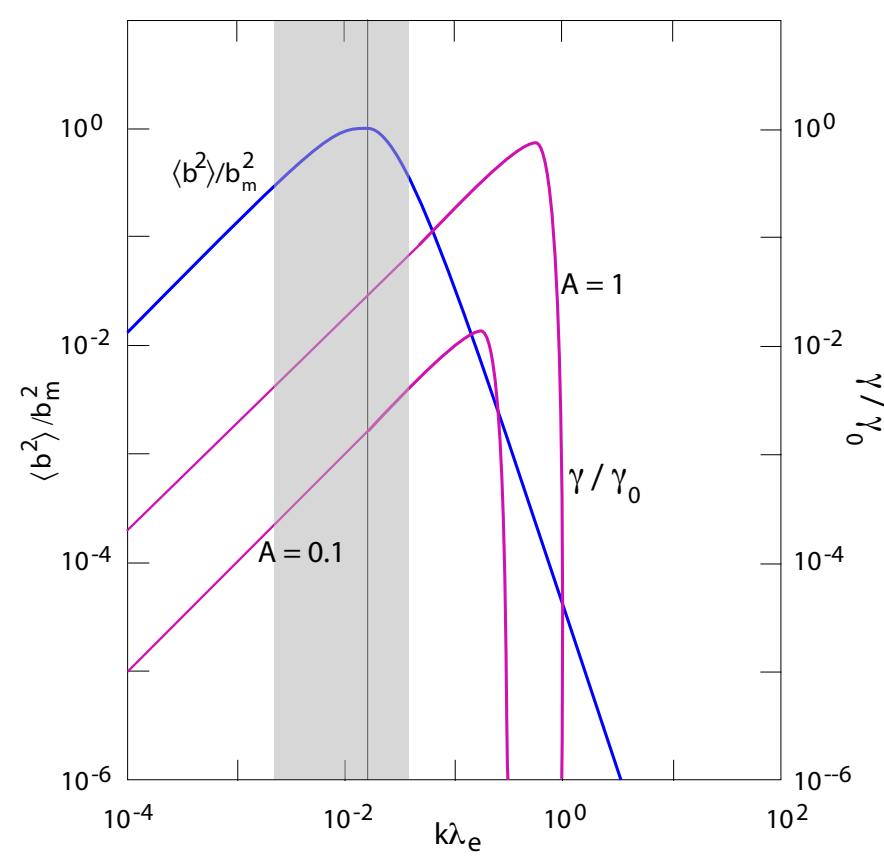

Fig. 2. Wavenumber dependencies of the normalised fluctuation spectrum and normalised growth rates. The normalisation of the thermal fluctuation spectrum is to its maximum value given in Eq. (13). Normalisation of the growth rate is to $\gamma_{0}=$ $\omega_{\mathrm{e}} \sqrt{\pi / 2}\left(c / v_{\mathrm{e}}\right)$. Since the growth rate depends on anisotropy $A$ it is given for the two cases $A=0.1,1$. Note the competition between growth rate and fluctuation level. At long wavelengths the high fluctuation level partially compensates for the low growth rate. The range of wavelength of interest in the magnetospheric tail is shown shaded. It centres around maximum thermal fluctuation level.

zero-frequency thermally-anisotropic Weibel mode can be written

$$
\frac{\left\langle|\boldsymbol{b}|^{2}\right\rangle_{k 0}}{\sqrt{2 \pi}}=\frac{\mu_{0}}{\omega_{\mathrm{e}}} \frac{c}{v_{\mathrm{e} \perp}} \frac{T_{\mathrm{e} \perp} k_{\perp} \lambda_{\mathrm{e}}(A+1)^{2}}{(A+2)\left[k_{\perp}^{2} \lambda_{\mathrm{e}}^{2}-A+m_{\mathrm{e}} / m_{\mathrm{i}}\right]^{2}}
$$

The 0-subscript refers to vanishing real frequency. Here the ion contribution has been retained. In the isotropic $A=0$ and Weibel-stable $-2<A<0$ cases, the spectral energy density vanishes at $k_{\perp} \rightarrow 0, k_{\perp} \rightarrow \infty$ and, in a proton-electron plasma, maximises at $k_{\perp} \lambda_{e} \approx 0.013$. Its maximum value is

$$
\left\langle|\boldsymbol{b}|^{2}\right\rangle_{k 0, m}=8.25 \times 10^{-23} \sqrt{\frac{T_{\mathrm{e}[\mathrm{eV}]}}{N_{\left[\mathrm{cm}^{-3}\right]}}} \quad \frac{\mathrm{V}^{2} \mathrm{~s}^{3}}{\mathrm{~m}}
$$

One might note that for positive anisotropies the current sheet is not in equilibrium anymore, and the thermal fluctuations explode close to the boundary of the unstable domain for $k_{\perp} \lambda_{\mathrm{e}} \sim \sqrt{A} \approx k_{0} \lambda_{\mathrm{e}}$ indicating onset of instability and phase transition.

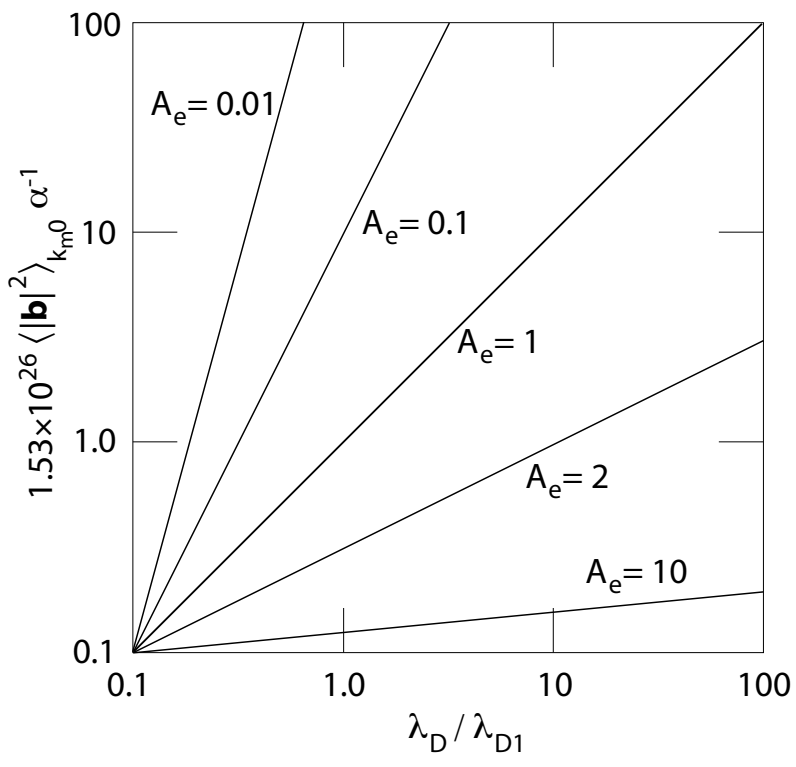

Fig. 3. Normalised thermal fluctuation level at maximum growing Weibel wave number $k_{\mathrm{m}}$ as function of Debye length $\lambda_{\mathrm{D}}$. The fluctuations are normalized to their value at $T_{\mathrm{e}}=1 \mathrm{eV}, N=1 \mathrm{~cm}^{-3}$. The Debye normalisation is taken to the Debye length $\lambda_{\mathrm{D} 1}$ at these numbers. As suggested by Fig. 2, the initial fluctuation level from where the maximum unstable Weibel mode grows decreases $\sim A^{-1}$ because of its dependence on $k_{\mathrm{m}}$, which increases as $\sqrt{A}$. Since the spectral energy density of fluctuations decreases $\sim k^{-3}$, the initial level of the fastest growing Weibel mode also decreases with growing anisotropy.

\subsection{Fastest growing Weibel mode}

The Weibel instability choses from this spectral energy density and supports the fastest growing wavenumber $k_{\perp \mathrm{m}}$. Inserting for $k_{\perp \mathrm{m}}$ the initial thermal level of the fastest growing mode becomes

$\left\langle|\boldsymbol{b}|^{2}\right\rangle_{k_{\mathrm{m}} 0} \simeq \frac{9 \mu_{0}}{4} \frac{m_{\mathrm{e}} c^{2}}{\omega_{\mathrm{e}}} \sqrt{\frac{\pi}{3} \frac{T_{\mathrm{e} \perp}}{m_{\mathrm{e}} c^{2}}} \frac{(A+1)^{2} /(A+2)}{\left(A-3 m_{\mathrm{e}} / 2 m_{\mathrm{i}}\right)^{2}}$

Since large thermal anisotropies are unrealistic, the cases of small $A \ll 1$ and large anisotropies $A \sim 1$ may be distinguished yielding the limiting initial levels

$\left\langle|\boldsymbol{b}|^{2}\right\rangle_{k_{\mathrm{m}} 0} \simeq \frac{\alpha \mu_{0}}{A} \frac{m_{\mathrm{e}} c^{2}}{\omega_{\mathrm{e}}} \sqrt{\frac{\pi}{3} \frac{T_{\mathrm{e} \perp}}{m_{\mathrm{e}} c^{2}}}, \quad A>\frac{3}{2} \frac{m_{\mathrm{e}}}{m_{\mathrm{i}}}$

with $\alpha=9 / 8$ for $A \ll 1$, and $\alpha=3$ for $A \lesssim 1$. Numerically:

$\left\langle|\boldsymbol{b}|^{2}\right\rangle_{k_{\mathrm{m}} 0} \approx 8.8 \times 10^{-28} \frac{\alpha}{A} \sqrt{\frac{T_{\mathrm{e}[\mathrm{eV}]}}{N_{\left[\mathrm{cm}^{-3}\right]}}} \frac{\mathrm{V}^{2} \mathrm{~s}^{3}}{\mathrm{~m}}$

where the temperature is measured in $\mathrm{eV}$, and the density is in $\mathrm{cm}^{-3}$. The numerical factor for the largest expected anisotropy $A \sim 1, \alpha=3$ is $\approx 2.63 \times 10^{-27}$. 


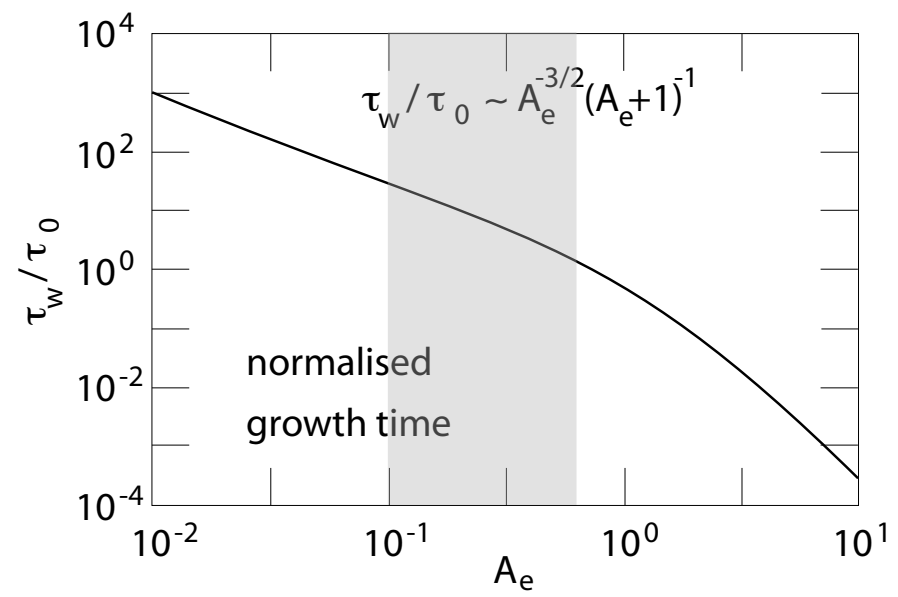

Fig. 4. Variation of the relevant growth times $\tau_{\mathrm{W}, \mathrm{m}}$ (Eq. 18) of the maximum growing Weibel mode with thermal anisotropy $A$ for maximum growth. The normalisation $\tau_{0}$ is a number which depends on $T_{\mathrm{e}}, N_{\mathrm{e}}$ and the value of the final magnetic field strength required for onset of reconnection. In the geomagnetic tail current sheet $\tau_{0} \gtrsim$ $0.1 \mathrm{~s}$. Even though the initial thermal level in Fig. 2 decreases with anisotropy, the times to reach a substantial Weibel field strength in the current sheet are mainly determined by the dependence of the maximum growth rate $\gamma_{\mathrm{m}}$ on $A$. They depend only logarithmically on the initial thermal wave field. They thus decrease with growing anisotropy. The shaded area shows the range of anisotropies and growth times expected in the geomagnetic tail current sheet.

The unstable Weibel spectral energy density evolves according to

$$
\left\langle\left|\boldsymbol{B}\left(t, k_{\mathrm{m}}, 0\right)\right|^{2}\right\rangle \approx\left\langle|\boldsymbol{b}|^{2}\right\rangle_{k_{\mathrm{m}} 0} \exp \left(2 \gamma_{\mathrm{W}, \mathrm{m}} t\right)
$$

The growth time of the fastest growing mode follows from this expression as

$\tau_{\mathrm{W}, \mathrm{m}} \approx \frac{1}{2 \gamma_{\mathrm{W}, \mathrm{m}}} \ln \frac{\left\langle\left|\boldsymbol{B}\left(k_{\mathrm{m}}, \tau_{\mathrm{W}, \mathrm{m}}\right)\right|^{2}\right\rangle}{\left\langle|\boldsymbol{b}|^{2}\right\rangle_{k_{\mathrm{m} 0}}}$

The spectral energy density of a $|\boldsymbol{B}|=1 \mathrm{nT}$ magnetic field fluctuation is $\left\langle\left|\boldsymbol{B}_{1 \mathrm{nT}}\right|^{2}\right\rangle_{k 0} \approx 4.3 \times 10^{-12} \mathrm{~V}^{2} \mathrm{~s}^{3} / \mathrm{m}$. This value may be used when estimating the time it needs for the maximum growing thermal-anisotropy driven Weibel mode in the magnetotail current sheet to grow up to a value comparable to the external (lobe) magnetic field $B_{0} \sim$ few nT. If we take the growth rate in the range $1 \lesssim \gamma_{\mathrm{W}, \mathrm{m}}<50 \mathrm{~Hz}$ which holds for $0.1 \lesssim A<1$, short growth times from thermal level to $1 \mathrm{nT}$ fields of the order of

$\tau_{\mathrm{W}, \mathrm{m}}>0.1 \mathrm{~s}$

are obtained, corresponding to mostly a few seconds of growth time in the magnetospheric tail. Given the uncertainty of the numerical values used, this is not an unreasonable estimate of the length of the ignition phase that initiates

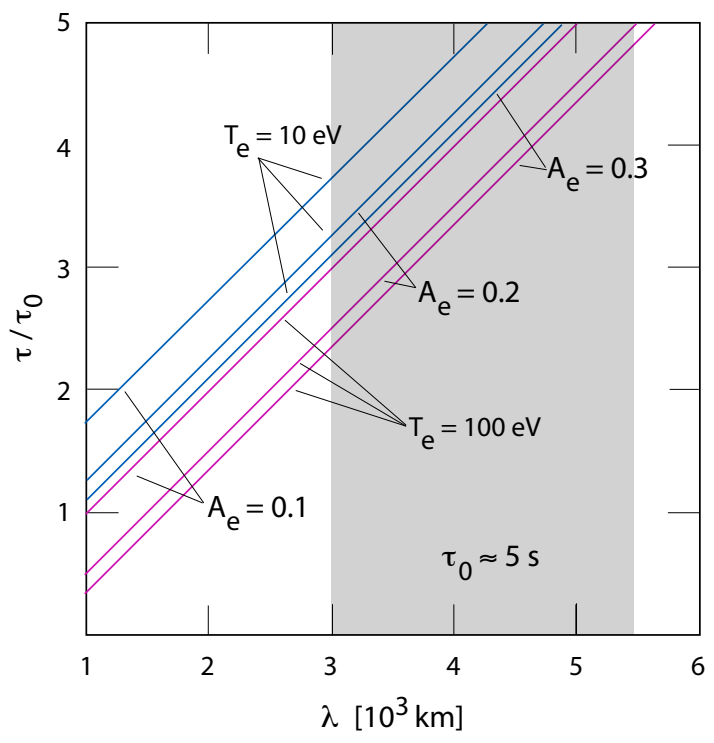

Fig. 5. The Weibel mode at long wavelengths: Growth time dependence on wavelength in the long wavelength range. The normalisation $\tau_{0} \approx 5 \mathrm{~s}$ is obtained for $T_{\mathrm{e}}=100 \mathrm{eV}, N_{\mathrm{e}}=1 \mathrm{~cm}^{-3}$, and $A=0.1$. Growth times are shown for two different temperatures $T_{\mathrm{e}}=10 \mathrm{eV}$ and $T_{\mathrm{e}}=100 \mathrm{eV}$ and three values of the anisotropy $A=0.1,0.2,0.3$. The shaded area is the acceptable wavelength domain for the geomagnetic tail.

reconnection in the tail current sheet, i.e. the time to produce initial X-points which subsequently start reconnection. Typical times for the evolution of substorms following onset of reconnection range from minutes to few tens of minutes and depend on the connection of the magnetotail reconnection site to the response of the ionosphere.

\subsection{Long wavelength Weibel modes}

Fastest growth corresponds to very short wavelengths $k_{\perp} \lambda_{\mathrm{e}} \lesssim \sqrt{A / 3}$. There may, however, as well be competition between decreasing growth rate and increasing initial fluctuation level at long wavelengths as shown in Fig. 2.

Equation (12) suggests that the spectral energy density of thermal fluctuations for $k_{\perp}^{2} \lambda_{\mathrm{e}}^{2}<A+m_{\mathrm{e}} / m_{\mathrm{i}}$ increases as $\sim k_{\perp} \lambda_{\mathrm{e}}$. In isotropic plasma $A=0$ this implies wavelengths $\lambda \gg 2 \pi \lambda_{\mathrm{e}} \sqrt{m_{\mathrm{i}} / m_{\mathrm{e}}} \approx 300 \lambda_{\mathrm{e}}$. In the magnetospheric tail the wavelength of maximum thermal fluctuation level is thus $\lambda \sim 1500 \mathrm{~km}$. The spectral energy density in this longwavelength range is given by Eq. (13).

At such wavelengths one can neglect the term $k_{\perp} / k_{0}$ in the expression for the Weibel growth rate. For small anisotropies $A<1$ the growth rate becomes

$\gamma_{W} \simeq \frac{4 A c}{\lambda} \sqrt{\frac{\pi T_{\mathrm{e} \perp}}{m_{\mathrm{e}} c^{2}}}$ 
Inserting the long-wavelength restriction on $\lambda$ yields

$\gamma_{W} \ll 0.01 A \sqrt{\frac{\pi T_{\mathrm{e} \perp}}{m_{\mathrm{e}} c^{2}}} \omega_{\mathrm{e}} \approx 1.4 A \sqrt{T_{\mathrm{e}[\mathrm{eV}]} N_{\left[\mathrm{cm}^{-3}\right]}} \mathrm{Hz}$

The Weibel growth rate, for $A \sim 0.1$ and $T_{\mathrm{e}} \sim 0.1 \mathrm{keV}$, in this wavelength range is thus of the order of $\gamma_{W} \sim 0.1 \mathrm{~Hz}$, one order of magnitude less than at maximum growth, yielding exponentiation times $\gamma_{W}^{-1} \sim 1 \mathrm{~s}$ and growth times $\tau_{W} \sim 10 \mathrm{~s}$ (see Fig. 5). This is the time a Weibel wavelength of $\lambda \sim$ $1500-3000 \mathrm{~km}$, i.e. roughly half one Earth radius, needs to grow from thermal level to an amplitude of $1 \mathrm{nT}$ in the geomagnetic tail prior to onset of reconnection.

In anisotropic plasma $A \neq 0$ and we may relax the condition on the wavelength. In this case the mass ratio in the thermal fluctuation expression becomes unimportant for reasonably large $A \gg m_{\mathrm{e}} / m_{\mathrm{i}}$. Then long wavelengths imply that $k_{\perp} \lambda_{\mathrm{e}} \ll \sqrt{A}=k_{0} \lambda_{\mathrm{e}}$ and

$$
\begin{aligned}
\left\langle|\boldsymbol{b}|^{2}\right\rangle_{k 0} & \simeq \frac{\mu_{0} m_{\mathrm{e}} c^{2}}{\omega_{\mathrm{e}}} \sqrt{\frac{\pi T_{\mathrm{e} \perp}}{m_{\mathrm{e}} c^{2}}} \frac{k_{\perp} \lambda_{\mathrm{e}}}{A^{2}} \\
& \ll \frac{\mu_{0} m_{\mathrm{e}} c^{2}}{\omega_{\mathrm{e}}} \sqrt{\frac{\pi}{A^{3}} \frac{T_{\mathrm{e} \perp}}{m_{\mathrm{e}} c^{2}}} \\
& \approx 3.2 \times 10^{-24} A^{-\frac{3}{2}} \sqrt{T_{\mathrm{e}[\mathrm{eV}]}} \quad \frac{\mathrm{V}^{2} \mathrm{~s}^{3}}{\mathrm{~m}}
\end{aligned}
$$

With $T_{\mathrm{e} \perp}=100 \mathrm{eV}$, and $A=0.1$ and using the former expression for the growth rate, one correspondingly expects growth times from thermal level of the order of $\tau_{W} \sim 100 \mathrm{~s}$, between 1 and $2 \mathrm{~min}$, for wavelengths of the order of $\lambda \sim 1000 \mathrm{~km}$ $\gg 2 \pi \lambda_{\mathrm{e}} / \sqrt{A} \sim 110 \mathrm{~km}$.

\section{Collisionless reconnection scenario}

These estimates are sufficiently encouraging for developing a microscopic scenario for collisionless reconnection as follows: Assume a plane homogeneous Harris current layer $J_{y}=-J_{0} \operatorname{sech}^{2}(2 z / \Delta)$, with $\Delta$ the layer half-width, separating two (lobe) regions of antiparallel magnetic fields. The magnitude of the field changes as $B_{\mathrm{X}}(z)=B_{0} \tanh (2 z / \Delta)$. Let this current layer be (locally) compressed until its width shrinks to $\Delta \sim \lambda_{\mathrm{i}}$. In the ion-inertial region the ions become locally non-magnetic and are accelerated in $-\hat{y}$ direction by the cross-field electric potential, carrying the pure ion Harris current. Electrons remain magnetised, transporting the magnetic field with inward velocity $-E / B(z)$ thus giving rise to Hall currents (Sonnerup, 1979) which are restricted solely to the ion-inertial region and close along the magnetic field lines which connect them to the ionosphere (Treumann et al., 2009). The $z$-dependence of the Hall currents is

$$
J_{\mathrm{H}}(z) \approx \frac{e N_{0} E}{2 B_{0}} \frac{\left[1-\Theta\left(|z|-z_{i}\right)\right] \Theta\left(|z|-z_{e}\right)}{\sinh (4 z / \Delta)}
$$

where $z_{\mathrm{i}, \mathrm{e}} \equiv \xi_{\mathrm{i}, \mathrm{e}} \lambda_{\mathrm{i}, \mathrm{e}}$ and $1 \lesssim \xi_{\mathrm{i}, \mathrm{e}} \in \mathrm{R}$ are rational numbers close to but larger than unity. Field line bending in reconnection is not taken into account here. Hall currents vanish in the centre of the current sheet at distances $|z| / \xi_{\mathrm{e}} \leq \lambda_{\mathrm{e}}$, less than the electron inertial length $\lambda_{\mathrm{e}}=c / \omega_{\mathrm{e}}$ where the electrons demagnetise. For the onset of reconnection the Hall currents are thus of no importance.

The non-magnetic electrons in the central current sheet experience the cross-field potential $\Delta U$, accelerate in $+\hat{y}$ direction and become the primary carriers of the cross-tail current here. Accelerated to large cross-tail velocities $V_{\mathrm{e}}>v_{\mathrm{e}}$, these electron currents excite the Buneman two-stream instability on growth times shorter than $\tau_{\mathrm{B}}<10^{-3} \mathrm{~s}$, a number holding in the magnetotail. The Buneman instability stabilizes within $0.01<\tau<0.1 \mathrm{~s}$ by heating the trapped electrons along $\pm \hat{y}$ until $v_{\| e} \sim V_{\mathrm{e}}$. As a consequence the current sheet electrons develop a positive temperature anisotropy $0<A<1$ which is sufficiently large to drive the Weibel mode unstable and result in the generation of a stationary magnetic Weibelfield $\boldsymbol{B}_{\mathrm{W}}=\left(B_{\mathrm{x}}, 0, B_{\mathrm{Z}}\right)$ in the current sheet with components in the $(x, z)$-plane perpendicular to both, the current flow and anisotropy directions. The fastest growing wavelength is $\lambda_{\mathrm{m}} \sim 2 \pi \lambda_{\mathrm{e}} \sqrt{3 / A}$.

In the magnetospheric tail current layer we have $\lambda_{e} \approx$ $5.4 / \sqrt{N_{\left[\mathrm{cm}^{-3}\right]}} \mathrm{km}$. The maximum growing wavelength of the Weibel magnetic vortices thus becomes shorter than $\lambda_{m}<$ $180 \mathrm{~km}$, the value obtained for a weak anisotropy $A \sim 0.1$. The time for this field to grow to values of the order of $1 \mathrm{nT}$ (or a fraction of it) is of the order of few seconds.

It is usually claimed that the Weibel instability stabilizes when the electron gyroradius in the Weibel magnetic field becomes comparable to the Weibel wavelength. When the electrons are accelerated to $>\mathrm{keV}$ energies their gyroradius in a $1 \mathrm{nT}$ magnetic field becomes the order of $\sim 100 \mathrm{~km}$, roughly the same order as the above estimated maximum wavelength. Thus the short wavelength Weibel field has sufficient time to grow to substantial values until it stabilizes self-consistently by deflecting the current electrons. Prior to this the Weibel field has penetrated the current sheet forming vortices and vertexes which serve as seed- $X$ points for reconnection which may then proceed at about along the lines that were discussed long ago in an attempt of formulating a kinetic theory of collisionless reconnection by ad hoc imposing a $B_{\mathrm{Z}}$-field on the current layer (Galeev and Zelenyi, 1975; Sagdeev, 1979). This attempt led to the proposal of a scenario for the spontaneous onset of magnetospheric substorms. In the presence of $B_{\mathrm{Z}} \neq 0$ the current sheet is in a metastable state that goes spontaneously unstable. The main deficiency of this theory was the lack of any reason for the appearance of $B_{\mathrm{Z}}$. Imposing it ad hoc is the equivalent of igniting reconnection artificially.

What happens in the long wavelength regime? Here we have $\lambda \gtrsim 300 \lambda_{\mathrm{e}} \approx 1.5 \times 10^{3} \mathrm{~km}$. The growth time to observable/relevant amplitudes we found to be of the order of 

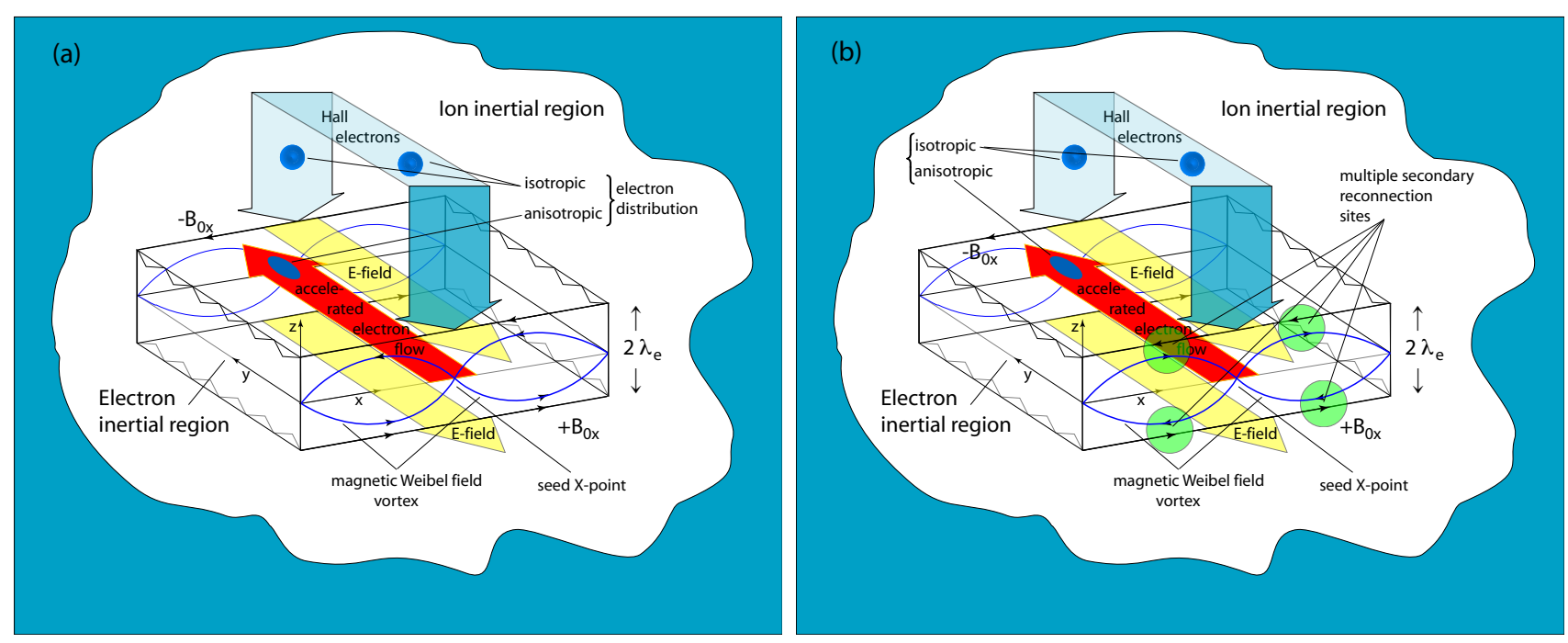

Fig. 6. Sketch of the electron-inertial region (width $\Delta z=2 \lambda_{\mathrm{e}}$ ) around the centre of the current layer, embedded into the ion-inertial region. Hall-electrons carrying the Hall-current in the ion-inertial region enter the electron-inertial region (shown on one side of the current layer only) with isotropic temperature distribution, experience the electric field $\boldsymbol{E}$, accelerate into $+\hat{y}$ direction. After being heated by the twostream instability they develop a temperature anisotropy and excite magnetic Weibel-vortices (blue) along $\hat{x}$ the vertexes of which serve as seed-X points for reconnection. The condition that $B_{\mathrm{Z}}=0$ at $z= \pm \lambda$ e allows for two types of Weibel vortices: (a) a symmetric (magnetically continuous) vortex-mode (same direction as the external fields $\boldsymbol{B}_{0 x}$ ), and a (b) anti-symmetric non-continuous vortex-mode (antiparallel to the external field) which yields tail-current bifurcation.The anti-symmetric mode gives rise to multiple reconnection sites (shown in green colour) and presumably leads to explosive reconnection.

$\tau_{W} \sim 10 \mathrm{~s}$, which is not unreasonable for the processes going on there.

The short wavelength modes grow about ten times faster than the long wavelength modes but may not be of substantial importance for reconnection until they cascade inversely down to longer wavelength structures. This could be provided by the coalescence of magnetic islands which is strongest at short wavelengths. The Weibel instability in this case excites an entire spectrum of magnetic field structures in the current layer. In the geomagnetic tail both the short Weibel modes and the long wavelengths modes provide seed $\mathrm{X}$-points on geophysically reasonable spatial and temporal scales.

\section{Discussion and conclusions}

The present approach has so far only implicitly taken into account the narrow width of the non-magnetic central current region which imposes boundary conditions on the evolution of the Weibel mode. Continuity of $\boldsymbol{B}$ at $z \sim \pm \lambda_{\mathrm{e}}$ implies a vanishing $B_{\mathrm{Z}}$ here. As demonstrated, this imposes limits on wave number and propagation angles of the Weibel mode. In addition the presence of a boundary implies that $B_{\mathrm{x}}$ is either parallel or antiparallel. Clearly the parallel case is preferred as the antiparallel case generates small-scale current bifurcation. On the other hand, this is possible because the evolution of the Weibel mode is completely independent of the presence of the external field.
One thus distinguishes between two types of Weibel modes depending on their propagation directions $\pm \hat{x}$. One of them (Fig. 6a) just causes seed-X points, the other (bi- or trifurcated) mode may lead to multiple - probably explosive - reconnection (see Fig. 6b). The reason for an explosive character lies in the fact that on the short scale across the boundary $z \sim \pm \lambda_{\mathrm{e}}$ between the inner (Weibel) current region and the external (Hall) region the electrons are unmagnetised such that the Weibel field can freely expand into $z$ until contacting the lobe magnetic field. This causes spontaneous reconnection between the contacting magnetic flux tubes and will force the inner current sheet to decay into a chain of highly dynamical magnetic islands (meso-scale plasmoids), as is immediately realised from Fig. $6 \mathrm{~b}$ when imagining the resulting magnetic field structure after multiple spontaneous reconnection has set on. In this case, the inner part of the current layer will become "turbulent" (multiply connected) on meso-scales the order of the Weibel wavelength. The inner current region decays into a "magnetic vortex street" consisting of (electronic) plasmoids and seed- $X$ points.

In principle, one may still worry why in both cases magnetic $X$ points will at all form even in presence of the Weibel mode. The reason for this is that the scale of the $X$ points is microscopic in the sense that neither electrons not ions are magnetised and thus are not affected anymore by the magnetic field. Vice versa, the magnetic field is independent of the presence of electrons on this scale; it behaves like a potential field in vacuum whose sources are outside the small box that contains the $\mathrm{X}$ point. This means that no electron 
currents flow here which are responsible for the anti-parallel fields! Because of this reason the contacting anti-parallel fields can like in vacuum freely rearrange their topology without affecting the particle components on the microscopic scale.

One may ask what structure of the field and current layer is expected in the direction parallel to the current flow. This question cannot be answered without detailed analysis. However, one may argue that the structure along the current is determined by two facts: the mechanism of electron heating, and the dynamics of the ions. Electron heating occurs in electron holes which have (short) longitudinal extension of $\Delta y \sim 100 \lambda_{\mathrm{D}}$, where $\lambda_{\mathrm{D}}$ is the Debye length. The heating scale is orders of magnitude longer including several to many phase space holes. However, though it is long, it is still microscopic. In the magnetotail current layer this length becomes the order of several $\sim 100 \mathrm{~km}$ to few $1000 \mathrm{~km}$ only. Hence, one suspects that the tail reconnection structure is inherently three-dimensional putting all two-dimensional models in question.

In addition, the present theory refers to stationary ions. The ions that carry the tail current move into direction $-\hat{y}$. Hence, the Weibel structures and the resulting reconnection sites as a whole move in the direction of the combined speed of the electron holes and current ions. Numerical simulations suggest (cf., e.g., Newman et al., 2001) that this direction is opposite to the electron flow velocity $\boldsymbol{V}_{\mathrm{e}}$, i.e. in the direction of ion flow. As a result one expects that the whole set of magnetotail-reconnection sites will displace slowly - roughly at translational velocity of the ion-sound speed $c_{\mathrm{s}} \simeq \sqrt{T_{\mathrm{e}} / m_{\mathrm{i}}} \sim 200 \mathrm{~km} / \mathrm{s}-$ into $-\hat{y}$-direction, the direction of ion flow, which in the magnetotail is westward. This is in accord with observation of the initial westward displacement of substorm sources. Mapping along the stretched magnetic field lines into the ionosphere decreases this translational westward drift speed by about one order of magnitude.

In conclusion, it is the thermal-anisotropy driven Weibel instability which provides the magnetic field to penetrate the inner region of the current layer, generates a local normal field component $B_{\mathrm{Z}}$ and, by producing short wavelength magnetic vortices and vertexes, it may ignite reconnection on a time scale of tens of seconds to few minutes in the magnetotail. This is in rough agreement with observations, e.g. in the magnetotail, and is sufficiently short for initiating magnetospheric substorms. On the other hand, any collisionless reconnection theory based on generation of anomalous diffusivity is, probably, quite unrealistic for reasons of time-scales. Diffusion also has to overcome logical difficulties which are not easily resolved (cf., e.g., the discussion in Baumjohann et al., 2010).

From our considerations it can be concluded that initiating reconnection in two and three-dimensional full particle simulations (any magnetohydrodynamic and multi-fluid simulations completely miss the physics of reconnection and can only be taken as a guidance for the macroscopically possi- ble magnetic field topologies) resolving the electron scales like those of Zeiler et al. (2002), Scholer et al. (2003) and others by artificially imposing seed-X-points is physically completely correct. Such simulations correctly mimic the action of the microscopic Weibel mode by bridging the initial phase of Weibel growth/saturation and replacing it by the final Weibel state. This is physically correct and reasonable. Such simulations can thus be used for investigating the further evolution of reconnection in a thin current sheet once seed-X-points have been generated by the Weibel instability. They can also be taken as starting points for investigating the subsequent behaviour of the various involved particle distributions, acceleration of particles and so on. Since, however, they can be performed only in rather small simulation boxes and with limited numbers of macro-particles (each of them standing for very large numbers of real particles) future research should concentrate on resolving the difficult problem of combining many such simulation boxes to build-up meso-scale models of reconnection which could be compared with observations. One might speculate that some kind of "renormalisation procedure" must be developed that conserves the important surviving (in the language of renormalisation group theory "relevant") effects in a small simulation box when going to larger scales.

Here we have presented a logically consistent chain of processes that provides a satisfactory mechanism for the spontaneous self-ignition of fast magnetic reconnection in thin plane and homogeneous collisionless current layers. Its numerical verification requires three-dimensional PIC simulations, resolving the fully electromagnetic electron dynamics in the current layer. A first step in this direction has been gone by Ricci et al. (2004) who initiated (collisionless) reconnection without imposing any magnetic islands on the current sheet. The reconnection starting in this case was attributed to the action of the lower-hybrid drift instability which naturally requires the presence of magnetised electrons (and thus holds for the region outside the electron inertial domain) respectively in the presence of a guide field, as in this case of simulations referred to. Guide-field reconnection is naturally quite different from the one considered in our model of non-guide field reconnection. However, when the guide field is directed parallel/antiparallel to the sheet current, electrons will be accelerated along the field in the way described by our model. They constitute a parallel beam which excites the Buneman instability, heat the electron component along the current and can indeed cause Weibel modes to grow. The simulation of Ricci et al. (2004) might have touched just this effect, in which case reconnection was caused by the action of the Weibel instability and was not initiated by anomalous diffusivity in the wake of the lower-hybrid instability.

We finally comment on the (real) observed reconnection in the magnetospheric tail. In fact, our model cannot be applied directly to the magnetospheric tail. It has been constructed for plane homogeneous current sheets. As noted above, the 
tail current sheet contains a residual component $B_{\mathrm{Z}}(x) \neq 0$ which is the uncompensated component of the geomagnetic quasi-dipole field. This component prevents complete demagnetisation of the tail electrons even in the centre of the tail current sheet, forcing the central electrons to perform a (slow) electric downtail drift. However, in this case a sufficiently strong guide field $\left|\boldsymbol{B}_{\mathrm{g}}\right|>B_{\mathrm{Z}}$ parallel to the tail current would substantially help as the electrons in its presence would become accelerated along $\boldsymbol{B}_{\mathrm{g}}$ by the cross-tail electric field in just the way noted in the previous paragraph, and the Weibel mechanism could set on. One might thus expect that imposing a guide field on the tail by some external mechanism, say during magnetospheric storms, would be crucial for initiating reconnection. One may also note that this type of guide field reconnection would possibly be responsible for the observed reconnection at the magnetopause where a guide field is naturally present.

Acknowledgements. This research is part of a Visiting Scientist Programme at ISSI, Bern, executed by RT. Hospitality of the ISSI staff is thankfully recognised, and the continuous encouragement by André Balogh, Director at ISSI, is highly appreciated. We gratefully acknowledge the referees' kind pointing our attention to the related papers by Zeiler et al. (2002) and particularly Ricci et al. (2004).

Topical Editor I. A. Daglis thanks P. Yoon and another anonymous referee for their help in evaluating this paper.

\section{References}

Baumjohann, W., Nakamura, R., and Treumann, R. A.: Magnetic guide field generation in collisionless current sheets, Ann. Geophys., 28, 789-793, doi:10.5194/angeo-28-789-2010, 2010.

Buneman, O.: Instability, turbulence, and conductivity in current-carrying plasma, Phys. Rev. Lett., 1, 8-9, doi:10.1103/PhysRevLett.1.8, 1958.

Buneman, O.: Dissipation of currents in ionized media, Phys. Rev., 115, 503-517, doi:10.1103/PhysRev.115.503, 1959.

Dungey, J.: Interplanetary magnetic field and the auroral zones, Phys. Rev. Lett., 6, 47-48, doi:10.1103/PhysRevLett.6.47, 1961.

Fried, B. D.: Mechanism for instability of transverse plasma waves, Phys. Fluids, 2, 337, doi:10.1063/1.1705933, 1959.

Fujimoto, M., Nakamura, M. S., Shinohara, I., Nagai, T., Mukai, T., Saito, Y., Yamamoto, T., and Kokubun, S.: Observations of earthward streaming electrons at the trailing boundary of a plasmoid, Geophys. Res. Lett., 24, 2893-2896, doi:10.1029/97GL02821, 1997.

Galeev, A. A. and Zelenyi, L. M.: Metastable states of diffuse neutral sheet and the substorm explosive phase, J. Exp. Teor. Phys. Lett. (JETP Lett.), 22, 170-172, 1975.

Nagai, T., Shinohara, I., Fujimoto, M., Hoshino, M., Saito, Y., Machida, S., and Mukai, T.: Geotail observations of the Hall current system: Evidence of magnetic reconnection in the magnetotail, J. Geophys. Res., 106, 25929-25950, doi:10.1029/2001JA900038, 2001.
Newman, D. L., Goldman, M. V., and Ergun, R. E.: Evidence for correlated double layers, bipolar structures, and very-lowfrequency saucer generation in the auroral ionosphere, Phys. Plasmas, 9, 2337-2343, doi:10.1063/1.1455004, 2001.

Øieroset, M., Phan, T. D., Fujimoto, M., Lin, R. P., and Lepping, R. P.: In situ detection of collisionless reconnection in the Earth's magnetotail, Nature, 412, 414-417, doi:10.1038/35086520, 2001.

Parker, E. N.: Sweet's mechanism for merging magnetic fields in conducting fluids, J. Geophys. Res. 62, 509-520, doi:10.1029/JZ062i004p00509, 1958.

Ricci, P., Brackbill, J. U., Daughton, W., and Lapenta, G.: Collisionless magnetic reconnection in the presence of a guide field, Phys. Plasmas, 11, 4102-4114, doi:10.1063/1.1768552, 2004.

Sagdeev, R. Z.: The 1976 Oppenheimer lectures: Critical problems in plasma astrophysics. I. Turbulence and nonlinear waves, II. Singular layers and reconnection, Rev. Mod. Phys., 51, 1-20, doi:10.1103/RevModPhys.51.11, 1979.

Scholer, M., Sidorenko, I., Jaroschek, C. H., Treumann, R. A., and Zeiler, A.: Onset of collisionless magnetic reconnection in thin current sheets: Three-dimensional particle simulations, Phys. Plasmas, 10, 3521-3527, doi:10.1063/1.1597494, 2003.

Scudder, J. D., Holdaway, R. D., Glassberg, R., and Rodriguez, S. L.: Electron diffusion region and thermal demagnetization, J. Geophys. Res., 113, A10208, doi:10.1029/2008JA013361, 2008.

Sitenko, A. G.: Electromagnetic Fluctuations in Plasma, Academic Press, New York, 1967.

Sonnerup, B. U. Ö.: Magnetic Field Reconnection, in: Solar System Plasma Physics, Vol III, pp. 45-108, edited by: Lanzerotti, L. T., Kennel, C. F., and Parker, E. N., North-Holland, New York, 1979.

Sweet, S.: The neutral point theory of solar flares, Proceed. IAU Symp., 6, 123-134, 1958.

Treumann, R. A. and Baumjohann, W.: Advanced Space Plasma Physics, Imperial College Press, London, 1997.

Treumann, R., Jaroschek, C. H., and Pottelette, R.: Auroral evidence for multiple reconnection in the magnetospheric tail plasma sheet, Europhys. Lett., 85, 49001, doi:10.1209/02955075/85/49001, 2009.

Weibel, E. S.: Spontaneously growing transverse waves in a plasma due to an anisotropic velocity distribution, Phys. Rev. Lett., 2, 83-84, doi:10.1103/PhysRevLett.2.83, 1959.

Yoon, P. H.: Spontaneous thermal magnetic field fluctuations, Phys. Plasmas, 14, 064504-064504-4, doi:10.1063/1.2741388, $2007 \mathrm{~b}$.

Yoon, P. H. and Davidson, R. C.: Exact analytical model of the classical Weibel instability in a relativistic anisotropic plasma, Phys. Rev. A, 35, 2718-2721, doi:10.1103/PhysRevA.35.2718, 1987.

Zeiler, A., Drake, J. F., and Rogers, B. N.: Magnetic reconnection in toroidal $\eta_{i}$ mode turbulence, Phys. Rev. Lett. 84, 99-102, doi:10.1103/PhysRevLett.84.99, 2000.

Zeiler, A., Biskamp, D., Drake, J. F., Rogers, B. N., Shay, M. A., and Scholer, M.: Three-dimensional particle simulations of collisionless magnetic reconnection, J. Geophys. Res. A, 107, 1230, doi:10.1029/2001JA000287, 2002. 